\title{
ARTICLE
}

\section{Bespoke ocular prostheses}

\author{
Sébastien Ruiters $\mathbb{D}^{1} \cdot$ Stéphan de Jong ${ }^{1} \cdot$ Ilse Mombaerts $^{1}$
}

Received: 22 August 2018 / Revised: 15 January 2019 / Accepted: 31 January 2019 / Published online: 5 March 2019

(c) The Royal College of Ophthalmologists 2019

\begin{abstract} prosthesis.

Methods Retrospective analysis of case series. corneal graft, and sclerocornea was reproduced. the success of rehabilitative prosthetic treatment of the patient.

\section{Introduction}

The cosmetic rehabilitation of patients with anophthalmia and microphthalmia involves the design of an ocular prosthesis. In particular, prostheses based on acrylic-resin allow adequate anatomic and aesthetic fittings [1-3]. As patients with an ocular prosthesis prefer their face as seen in a mirror, the aesthetic result is best assessed using mirrors instead of photographs [4]. Herein we report a series of patients with ocular prosthesis tailored to the specific conditions of the anophthalmic/microphthalmic socket and contralateral eye.
\end{abstract}

Purpose To report the customized approach of patients with anophthalmia or microphthalmia with bespoke ocular

Results The study included cases with anophthalmia with upper eyelid deformity (one patient), microphthalmia and contralateral corectopia (one patient), microphthalmia with contralateral corneal graft (one patient), and congenital clinical anophthalmia with contralateral sclerocornea (one patient). Using techniques of embedded autologous hair and coating of adhesive pigment emulsion in the ocular prosthesis, the physical appearance of, respectively, an upper eyelid, corectopia,

Conclusion Tailoring the ocular prosthesis to the distinct condition of the anophthalmic socket and contralateral eye adds to

\section{Case reports}

\section{Case 1}

A 25-year-old female suffered from loss of the right eye and full-thickness marginal defect of the right upper eyelid following a bomb explosion 3 years before (Fig. 1a). We recreated a mock eyelid integrated in the prosthesis. The

Sébastien Ruiters

sebastien.ruiters@uzleuven.be

1 Department of Ophthalmology, University Hospitals Leuven, Herestraat 49, 3000 Leuven, Belgium appearance of skin within the prosthesis was emulated with dark brown pigment similar to the skin colour of the patient. To resemble an eyelid margin with lashes, a thin stripe of black pigment and small pieces of autologous brow hair were added at the lower level of the artificial skin area, all of which were embedded in the resin of the prosthesis through coating of blended pigment powder and liquid resin (Monopoly, Factor II inc., USA) (Fig. 1b). The volume of the prosthesis was enlarged to enable its retention within the anophthalmic socket. Both the prosthesis and remaining upper eyelid had limited range of motion (Fig. 1c).

\section{Case 2}

A 70-year-old man, with bilateral congenital corectopia, had anophthalmos of the left eye secondary to a perforating injury at the age of 10 years old. The patient was unhappy with the "normal" prosthetic eye for the different physical appearance with his seeing eye (Fig. 2a). For this reason, we designed a prosthesis with an off-centre pupil that matched the corectopia of the contralateral eye (Fig. 2b).

\section{Case 3}

A 47-year-old man with anterior segment dysgenesis due to Axenfeld-Rieger syndrome had a history of bilateral glaucoma tube-shunt surgery and penetrating keratoplasty, of which the right eye evolved to phthisis with esotropia 


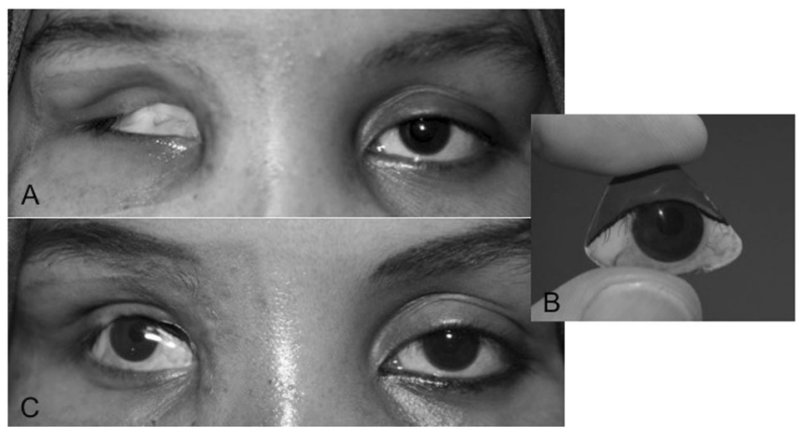

Fig. 1 a Case 1, with posttraumatic anophthalmos with upper eyelid defect at the right site. b Ocular prosthesis with embedded artificial upper eyelid. c Same patient with the prosthesis

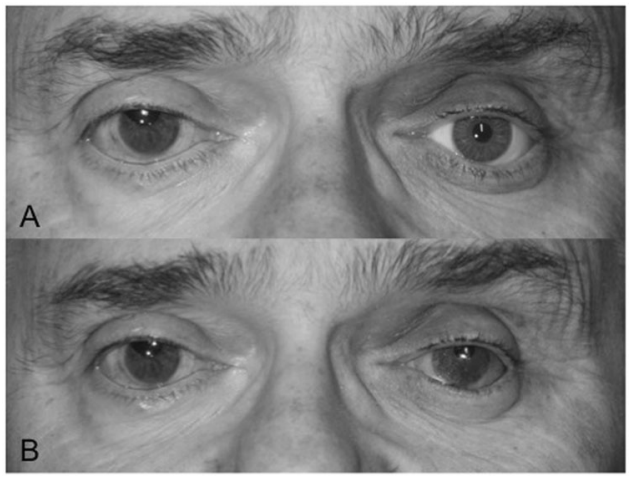

Fig. 2 a Case 2, showing corectopia of the right eye and left prosthesis with middle-centred pupil. b Same patient with prosthesis with corectopia

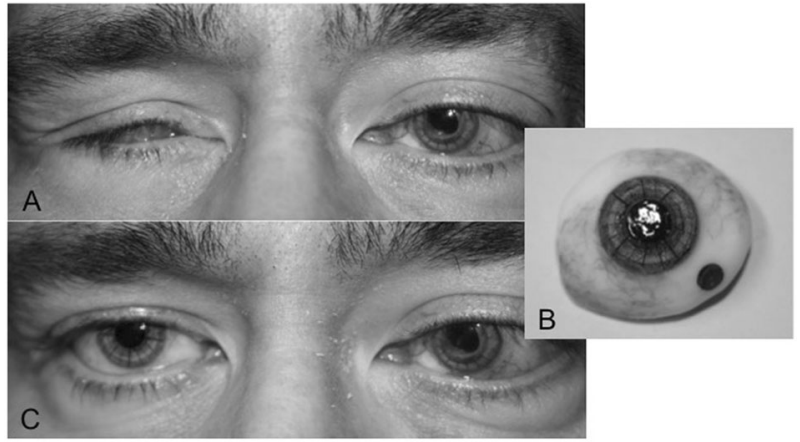

Fig. 3 a Case 3, right esotropic phthisis eye from developmental anomaly. b Frontal view of the ocular prosthesis with an artificial corneal graft. The black dot at the inferior part of the prosthesis, to allow the patient to identify bottom from top, is made larger then normal for reason of poor vision of the patient. c Same patient wearing the prosthesis on the right site

(Fig. 3a). The patient requested an ocular prosthesis that resembled the eye prior to the phthisis. To mimic corneal graft sutures, we embedded small pieces of autologous scalp hair at the level of the iris in the resin of the prosthesis (Fig. 3b) [5]. The iris-pupil-complex of the prosthesis was

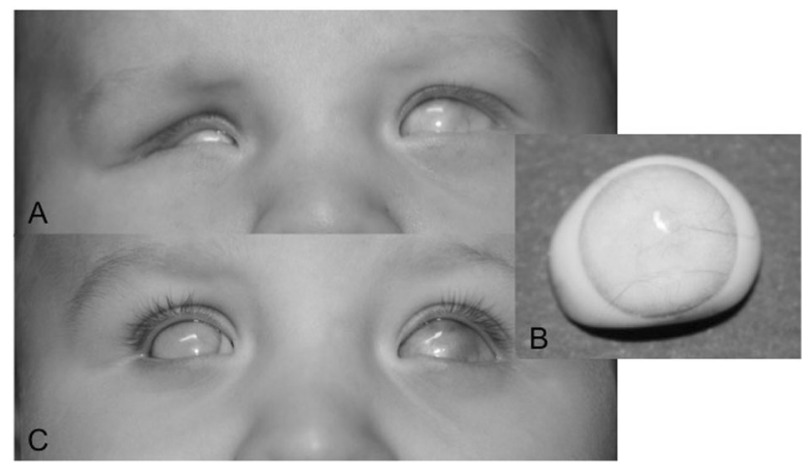

Fig. 4 a Case 4, clinical anophthalmos of the right site with sclerocornea of the left eye. b Frontal view of the ocular prosthesis with artificial sclerocornea. c Same patient with the prosthesis at the right site

positioned in relation to the eyelid fissure in such a fashion to obtain orthotropia (Fig. 3c).

\section{Case 4}

A 1-year-old boy with mutation of the sex determining region Y-box 2 (SOX2) gene had congenital clinical anophthalmia of the right eye and severe sclerocornea of the left eye. There was no light perception in both eyes and visual prognosis was poor. To stimulate orbital growth, we fitted scleral shells with increasing size at regular intervals in the right socket (Fig. 4a). To create the appearance of a sclerocornea, a thin layer of resin with white pigment was added anterior to the blue-coloured iris. The corneal vessels were reproduced, similar to the standard method for scleral vessels, with fluffs of red embroidering thread (Fig. 4b, c).

\section{Discussion}

In this study we present a series of four patients with severe physical eye defects and compromised vision and appearance, in whom symmetry with the contralateral eye was achieved with bespoke ocular prostheses. Case 1 highlights the potential to recreate the appearance of an eyelid with the ocular prosthesis as an alternative to a facial epithesis. Although not giving a skin feel as with silicone, the acrylic eyelid integrated in the ocular prosthesis was preferred to a silicone eyelid prosthesis because of the higher durability and superior retention of hair [6]. Case 1 and 3 introduce the use of autologous hair in an ocular prosthesis to reproduce, respectively, eyelashes and corneal sutures as alternative to synthetic fibres [7]. Case 2, 3 and 4 show the possibility to customize the shape and colour of the artificial cornea, iris and sclera to the microphthalmic socket and contralateral eye [8]. Although possibly used in common practice, to the best 
of our knowledge, such a prosthetic approach tailored to distinct eye conditions has not been described in the literature.

\section{Summary}

\section{What was known before}

- A bespoke ocular prosthesis aims to restore facial symmetry combined with comfortable wear.

- The fabrication of an ocular prosthesis is an artisanal process performed by an ocularist.

\section{What this study adds}

- This study highlights the importance of a customized approach in prosthetic rehabilitation and describes a tailored technique using pigment and autologous hair to design an ocular prosthesis in patients with altered anatomy of the anophthalmic socket or fellow eye.

Funding This research received no specific grant from any funding agency in the public, commercial or not-for-profit sector

\section{Compliance with ethical standards}

Conflict of interest The authors declare that they have no conflict of interest.

Publisher's note: Springer Nature remains neutral with regard to jurisdictional claims in published maps and institutional affiliations.

\section{References}

1. Bannwart LC, Goiato MC, dos Santos DM, Moreno A, Pesqueira AA, Haddad MF, et al. Chromatic changes to artificial irises produced using different techniques. J Biomed Opt. 2013;18:58002.

2. Kaltreider SA. The ideal ocular prosthesis: analysis of prosthetic volume. Ophthalmic Plast Reconstr Surg. 2000;16:388-92.

3. Jahrling R. The role of the ocularist. In: Migliori M, editor. Enucleation, evisceration and exenteration of the eye. Woburn: Butterworth-Heinemann; 1999. p. 13-76.

4. Mombaerts I, Missotten L. The patient with an ocular prosthesis and his mirror image. Ophthal Plast Reconstr Surg. 2011;27:343-7.

5. Pine K, Sloan B, Jacobs R. Clinical ocular prosthetics. New York: Springer; 2015.

6. Hatamleh M, Polyzois G, Nuseir A, et al. Mechanical properties and simulated aging of silicone maxillofacial elastomers: advancements in the past 45 years. J Prosthodont. 2016;25:418-26.

7. Avinash C, Nadiger R, Guttal S, et al. Orbital prosthesis: a novel treatment approach. Int J Prosthodont Restor Dent. 2012;2:19-23.

8. Goiato MC, Bannwart LC, Haddad MF, dos Santos DM, Pesqueira AA, Miyahara GI. Fabrication techniques for ocular prostheses: an overview. Orbit. 2014;33:229-33. 